\title{
Methods for investigating architecture: from the physical to the digital
}

\author{
D. Porter \& R. Hanna \\ The Mackintosh School of Architecture, Glasgow School of Art, UK
}

\begin{abstract}
This paper examines the relationship between traditional and modern methods of architectural investigation, namely: the 'sketch', the 'physical model' and the 'computer'. It aims to test two propositions put forward by two schools of thought regarding the architectural 'method'. The first suggests that the introduction of the computer as a working method would lead to the demise of the more traditional methods of sketching and model making. The second acknowledges the potential of technology but maintains that the computer as a device for abstraction is less effective than traditional systems of representation. A framework, based on the critical review of literature, was established and used as an intellectual vehicle for testing both propositions. It partly revolved around linking Popper's three worlds of knowledge, the subjective, the physical and the objective to the three variables: the sketch, the physical model and the computer. The study concludes that although computers are superior in dealing with complexity, design fixation, and performance analysis of design alternatives, this power does not constitute a sufficient condition, as a cause, for the demise of traditional working methods, as an effect to follow. On the other hand, the biggest strength of traditional methods of sketching and model making is not in the link between drawing and 'visual thinking', as purported by many authors, but it is the material sense of the 'physical' model that gives rise to other senses such as 'touch' and 'physicality'. The missing notion of physicality from both 3D objects and materials generated by the computer is undoubtedly its downfall. Keywords: the sketch, the physical model, architectural science, the computer, Popper, worlds of knowledge.
\end{abstract}

\section{Introduction}

There is growing evidence to suggest that the type of design media one works with will influence the manner in which design problems are explored and consequently determines the nature of architectural product. 
Wienands highlighted the value of 'languages: words, drawings and models' architects work with as a vehicle for architectural thought [1, pp. 8-10] suggesting that 'more differentiated environmental forms are the result of more differentiated thought processes and these require differentiated architectural languages. The more languages or methods used and the more often they are interchanged, the greater the insight gained. The interchange of languages is a methodical help'. A similar view was echoed by Heath who equates the ability of a designer to conceive ideas and produce solutions with the nature and power of conceptual 'tools' in her/his disposal [2]. He opined that the limitations of method constrain thinking and will be revealed as limitations of the design. 'The student who cannot draw freely will design within the limits of his power of representation. He is the victim of analogue take-over' [2]. However, traditional working methods using models and drawings were pre-eminent during the seventies where little knowledge existed on the use of computers, as 'symbolic' models, within the design process. Computers were expensive to acquire and CAAD programmes were not only limited in terms of three dimensional modelling capabilities but also difficult to use. Today it is a different picture as both hardware and software have improved markedly in terms of performance and modelling capabilities. Our knowledge about their integration in the design process has matured and become well established both in education and practice. In fact some of the complexity of the design process can only be dealt with effectively with the use of CAAD in the modelling as well as the manufacturing process of buildings. What is unique about physical models compared to symbolic models of computer and drawings is that notion of the 'physical state' which, as a basis for epistemology, is deep rooted in the 'empiricist' school of thought. Unlike the 'rationalist' movement, the empiricists argue that we are born ignorant, we are born without knowledge, and everything we learn is through our senses when they interact with the physical world.

\section{The architectural physical model}

The use of physical models within design process can serve two purposes: to help designers to 'explore' and develop design ideas at a conceptual level and to 'experiment' with design ideas, i.e. a vehicle for testing structures, acoustics, etc.

Moholoy-Nagy, an advocate of the use of architectural models in design at the Bauhaus, encouraged students to perform 'experiment in space' and create open 'constructions' in many materials such as wood, wire, cardboard and perspex so that they may arrive at spatial solutions in three dimensions that satisfy functionally and aesthetically [3]. Knoll and Hechinger classified architectural models into three categories: topographic (site models, landscape models); buildings (urban models, structural models, single building models and interior models); special (design models, object models). These models can be developed in three stages relating to stages of the design process: conceptual, developmental and presentation [4].

The significance of both models and drawings was examined in an exhibition at the Institute of Architecture and Urban Studies, New York in 1976. The aim of the exhibition 'Idea as Model' according to Popper was to 'clarify new means of 
investigating architecture in three dimensions' and regard models as 'studies of a hypothesis, a problem, or an idea of architecture' [5]. Popper went on to make a significant distinction between modern architecture and the architecture of Beaux-Art in terms of philosophy in relation to their pre-eminent working methods. He draws on Drexler's argument during an exhibition at the Museum of Modern Art the year before. Drexler maintained that modern architecture in its drive towards objectivity relied on the physicality of materials in models as an expression of 'reality' whereas 'the freer, fictive architecture of the Beaux-Arts had depended on the illusions of drawings' [5]. Sharing Drexler's views. Stern argued that the Modern Movement, in an attempt to undermine other types of architecture such as the Beaux-Arts, supported drawing styles which were 'neutral' in comparison to the Beaux-Arts' 'rendered' drawings as well as showing a preference for physical models over drawings [6]. Perhaps the Modern Movement was preoccupied with two notions: how to base architectural knowledge on 'rational' thinking and how to create 'ideas in the objective sense'. Also, the Modern Movement was and is still immensely influenced by Popper's pluralistic philosophy that the world of knowledge, which architecture is part of, is comprised of three ontologically distinct sub-worlds. The first is the physical or the world of physical states or 'visibilia', i.e. materials. The second is the 'mental' or the world of mental states. In architecture this world could be that of design ideas in the architect's mind before they become representations through words, diagrams and sketches. Popper's third world concerns the world of 'intelligibilia', or 'objective ideas'. This world of scientific and mathematical theories is of great importance as it has a great influence on the first world [7]. The world of computers today is part of this world as it based upon artificial intelligence which emanated from scientific and mathematical logic. Computers as a way of thinking have an element of objectivity as they show the designer what is there and allow him to test with confidence his ideas in relation to environmental and structural sciences. The thesis that is emerging in this paper is that somehow, the sketch, the model and the computer each belongs to one of Popper's three worlds of knowledge. The intellectual discourse seems to suggest that the use of computers in design should be encouraged if we were to explore the immense potential of Popper's third world.

Eisenman, citing the role of photographs, argues that the conceptual essence of a 'model' is a 'drawing' and 'a photograph of a building is a narrative record of a fact... the reality of the model because it is the view which reveals its conceptual essence as an axonometric drawing' [5]. Perhaps the role of a photograph is to mediate between a drawing and a model. Yet, Eisenman's view of a photograph being a mere recording device only touches one functional aspect. Other aspects include being a device for demonstration, showing high levels of detail and as a measuring tool, i.e. test structural deflection under different loading conditions.

\subsection{The architectural 'science' model}

On science models in design, Steven argues that such models are an important vehicle to deal and cope with reality and when models are 'deficient' then we 
have to accept the lesser learning aid of 'rules of thumb' [8]. He calls on architectural science educators to 'impart even a little theory instead of spoonfeeding students wads of rules of thumb'. His criticism is based on two notions; that rules of thumb provide very little understanding and that they are often contradictory, incomplete and lacking in theory. Models can and have been used to test structural ideas in design. For example Corbusier tested the feasibility of his structural concepts through models. His structural concept for the Philips Pavilion at the Brussels International Fair, a complex hyperbolic paraboloid in pre-stressed concrete, was tested by Bouma through a plaster model to prove its feasibility $[9$, p. 19]. However, there seems to be claims that the structural concept of hyperbolic parabolic tensile tent came from Xenakis, a Greek architect and music composer who worked in Corbusier's office at the time. Xenakis's complex tensile parabolic form was to be constructed out of lightweight materials. For acoustical reasons the material was changed to prestressed concrete to provide acoustical mass for sound insulation. Xenakis's complex parabolic form was generated out of straight lines from pre-cast concrete panels [10]. Gaudi, whose 'poetry of form' gave architecture original thoughts and philosophy on meaning, initiated new methods of structural analysis and calculation using stereoscopic models built with cords and small sacks of pellets to simulate the design of Colonia Guell (1898-1916). A framework of strings was established by hanging them from points representing the specific location of columns in the plan. Sacks filled with pellets and the weight of each sack was scaled down by a factor of $1 / 10000-$ a fraction of what each arch would have to support [11]. This was an interesting moment in the history of architectural technology as Gaudi realised the importance of loading 'similitude' between the model and the prototype. In a seminar on structural model analysis, organised by Princeton University Architecture School, Billington et al. called for a principle of 'similitude'- reduction by a scale factorto be adopted if model analysis is to have any significance as a design tool for structures [12]. While most students use Billington's principle of 'geometric similitude', they pay little attention to the other two principles: material similitude and loading similitude. The material similitude implies that the modulus of elasticity for both the model and the prototype is the same. Candela, on the other hand, argues against the use of scale models for structural analysis of complex geometry, such as hyperbolic paraboloid, in favour of other method such as mathematical calculations. He maintains that 'some people claim that if the mathematics is too hard, we can always revert to the testing of scale models or photo elasticity' [13]. In such structures, Candela argues, the forces at the edges, which determine the size of edge member, are extremely important and the only way to determine these forces is through calculations. He questions the issue of materials used in models being always different from the real thing. $\mathrm{He}$ calls for the use of more precise symbolic models in design. An answer to the questions he raised is through the use of computer modelling of structural behaviour. Not only computer models are quicker in calculations than manual methods, but also they allow the designer to visualise flexes, deformations and stresses within structures which, in the long run, will improve the general 
understanding of structural behaviour. Citing the experiments of Otto on three dimensional models, Janke views the complexity of interaction between construction and design as a reason for not using conventional calculations on two dimensional sectional diagrams [1, p. 62].

The most common type of architectural science models in design is the light model where scaled physical models provide a relatively accurate simulation of daylight in the prototype. For a complete analysis on the advantages and disadvantages of using physical model to explore daylight see a recent paper by both authors [14]. On the thermal performance of buildings, models can be used for two purposes: the analysis of air movement around building using wind tunnel studies and the assessment of 'skin' changes on indoor temperature. While using models for wind studies is very well known and documented, little is known about using physical models to assess thermal performance. What a scale model offers is the opportunity to test changes in orientation, shading and fabric on indoor temperature under actual climatic conditions.

The viability of using scale models at $1 / 4$ and $1 / 9$ for thermal studies has been investigated by Alexander who concluded: 'the models... appear to provide a useful and practical means of observing the thermal response to climatic variations of different materials and forms of construction. Such observations are of value in assessing the suitability of particular forms of construction for particular climates' [15]. Drysdale built a number of thermal models for houses situated nearby and took measurement of temperature inside the models and the prototypes. He found that both temperatures were comparable. However, he had to make some adjustments to ventilation rates in the models [9, p. 149]. The last discipline in architectural science where models are under utilised is acoustics and in particular sound behaviour in an enclosure. Barron suggested that to 'reproduce an auditorium in a scale model is simpler than many other spaces, because most surfaces are acoustically hard and can be reproduced by hard smooth surfaces, such as varnished timber or plastic materials' [16]. A scale factor of 1:8 or 1:10 was recommended for models to be representative. However he raised many questions regarding the height of labs that can house such large models as well as expenses associated with constructing the models in the first place. Models at scale 1:50 were suggested with a small loss of accuracy.

\section{Drawing as an architectural idea}

The relationship between architecture and drawing has always been poetic and intense. More importantly, it is the relationship between 'design', regarded as the distilled essence for the discipline of architecture, and the 'sketch', that attracted a great deal of research and scholarship. For instance, Goldschmidt investigates the process of sketching and argued that by sketching the designer does not draw the images he recorded in his mind 'but creates visual displays which help induce images of the entity that is being designed' [17]. Goldschmidt went on to suggest that the 'dialectics of sketching' is the 'oscillation' of 'arguments' between two states until a design solution is reached. If the solution to a design problem is seen as a design 'hypothesis', then sketching can be the process 
whereby a shift between propositions and counter-propositions occur until a qualitative transformation in the design argument is reached. The type of knowledge used to resolve the conflict could be graphical or non graphical.

Further examination of how much of design can be regarded as sketching is needed. Certain phrases, such as 'the idea-sketch' imply that a significant part of design is sketching. Ziesel's definition of design draws attention to its complexity as an activity as it encompasses three sub activities: imaging, presenting and testing [18]. While the role of sketching in 'imaging' is less obvious, its role in judging, testing and refuting design hypothesis at the conceptual design phase and presenting design ideas is there to see. Presumably part of the problem is that at the conceptual design stage the images held in the 'mind' and the 'sketches' drawn are interchangeable and the continuous feedback which is interactive between the two, is the process at work where the images sketched are judged against those stored in the mind. Yet on further examination of literature the pioneering work of Rudolph Arnheim is very relevant to how 'sketching' and 'imaging' are related. On drawing as a form of representation, Arnheim's treatise on 'Art and Visual Perception' [19], warns against art being drowned by talk, remarking: 'visual things cannot be expressed in words' and 'verbal analysis will paralyse intuitive creation and comprehension'. In 'Visual Thinking' [20, pp. 1-13], he asserts that the separation between seeing/perceiving and thinking/reasoning is unreal and misleading. On the doctrine of 'imageless thought', Arnheim seems to argue against the idea that thinking is possible without images, a thought which also came out of Buhler's experimental studies, [20, p. 101] who maintains that thinking needs a media to happen which could be through words. He goes on to suggest that the imagery can happen below the level of consciousness which cannot be detected by subjects during psychological experiments. To extend the argument further to design, it seems architectural 'thinking' cannot happen without 'imaging', and 'sketching' and imaging are closely related since 'images' of an 'idea' have to undergo a process of pictorial reasoning through 'sketching' before the idea becomes a designed entity (an object) on paper. Other researchers, such as Goldschmidt [21] introduces the dimension of 'sketching' as the third dimension in visual perception in addition to Arnheim's two dimensions of thinking and imaging. Goldschmidt's famous phrase of 'figural conceptualisation' suggests a fusion between two things and a rejection to any dichotomy between 'concept' and 'figure', i.e. the 'idea' and the 'sketch'. She sees the activities involved in sketching as being: active sketching (hand) $\rightarrow$ passive perception (eye) $\rightarrow$ active cognition (brain). A further argument against the divide between 'concept' and 'sketch' is artificial, came from McKim who introduces 'idea-sketching' as an evidence for some degree of fusion between figural and mental processes at the early design stage [22]. According to McKim graphic 'ideation'- the generation of ideas through drawing- occurs through an iterative communication loop where ideas can be added, processed and modified by a collective action by the eye, the brain and the hand through the 'sketch'. Mezughi [23] identifies two levels of ideation at the conceptual design phase: strategic and tactical. The strategic can be regarded as gestational, aiming to 
develop visual scenarios and/or images that act like a thesis or anti-thesis before an induced image of the solution is reached and sketched on paper. The tactical level is the 'selection' or 'focus' on one final image or solution.

There seems to be others that place less emphasis on the importance of sketching on the act of visual thinking manifested through seeing and imagining. The implication is the link between sketching and the generation of ideas, is not as strong as previously thought. For example, Levens wrote 'one source of confusion in thinking about design is the tendency to identify design with one of its languages, drawing. This fallacy is similar to the confusion which would result if musical composition were to be identified with the writing of notes... Design like musical composition, is done essentially in the mind and the making of drawings or writing of notes is a recording process' [24]. The limitations of drawing as a recording device were exposed by Lotz who, after investigating the architectural drawings of the Renaissance, concluded that circular interiors could not be drawn 'to provide useful information, such as scaled dimensions' [25]. Moreover, Evans questioned the 'history of architecture' and the special importance it placed on drawing compared to other forms of representation. He raised serious concerns about the 'objectivity' of drawing as an intellectual system of architectural thought. He sought explanations for two intertwined questions: 'how architectural spaces arose out of the deployment of depthless designs, and how architectural space was drawn into depthless designs' [25].

\section{The symbolic world of computers: architecture with machines}

The computer offers the designer a world of knowledge different from both conventional drawing and the physical model. It is a world based on logic, mathematics, precision and artificial intelligence. When the designer's cognition interacts with the computer a dialogue begins between two systems of thought: the 'artificial' and the 'biological'. It is through this interaction that design cognition- receiving, manipulating and processing information, is bound to experience a change in the way it deals with design problems. This change can take many forms, for instance in designer's creativity domains- ideation fluency and variety [26], in his new attitude toward the design process 'before' and 'after' using the computer [27], in his ability to test design ideas in an objective sense [14]. Above all there would be a change in the designers 'visuality' and the way he sees and perceives images and the way the physical world is experienced through 'simulated' reality.

Review of literature reveals a gulf in opinion on computers and design. Some view the computer as a 'medium' for conceptual design while others regard it as a production 'tool' with little impact on design thinking. The gulf of opinion is not only between theoreticians but also between prominent practitioners. For example in his treatise 'new science=new architecture' Jencks calls for a departure from the old Newtonian linear science to other forms of science such as that of complexity, fractals and non-linear systems. Architecture as 'a form of cultural expression' has to have a similar shift in the framework of thought, he 
argues. He then cites three 'seminal' buildings of the 1990s to support his thesis of shift. Gehry's Bilbao, Eisenman's Aronoff Centre, Libeskind's Jewish Museum 'are three non-linear buildings and were partly generated by nonlinear methods including computer design', maintains Jencks. He goes on to question the role of metaphor in the three buildings and suggests that 'new science $=$ new language $=$ new metaphor' [28]. Against this is Frampton who advocates a strong link between architecture and building in the 'material' world. Digital design on the computer is a 'fantasy' unless it conforms to 'tectonic', material requirements of the physical world [29]. However, conformity to the 'material' world may inhibit the 'subjective' experimentation of minds in the 'objective' world of computers. A similar conflict on computers and design exists between two world-class architects. Eisenman's writing identifies two intellectual themes about computers and architecture. First, he highlights the challenges to architecture from the 'electronic paradigm' as 'reality' is defined through simulation and 'appearance' is valued over 'existence' [30]. Secondly he acknowledges the creative potential of computers as he asserts that 'the computer gives you the possibility of constructing objects that you would never do directly from the mind to the hand. We constantly produce models after having conceptualised them using the computer, a process of constant refinement' [31]. In contrast, Gehry remains skeptical about the computer's ability to design, stating 'the computer is a tool, not a partner, an instrument for catching the curve, not for inventing it' [32]. However, recent progress in software engineering has furthered the capabilities of some CAAD packages and increased their creative potential as a conceptual tool at an early design stage. In programmes like Rhino, a NURBS modeller, 3D free form organic surfaces and solids can be created intuitively at the early design stage, overcoming serious limitations of traditional polygon software. This appears to address the issue of orthogonal rigidity of the Cartesian system, criticised by Gomez for 'representing another form of modernistic rationality' [33].

Recent work on genetic programming may produce new 'evolutionary' CAD tools that can help designs to evolve from scratch through a process of mutation [34]. Bentley suggests that evolutionary CAD tools, 'allow the designer to explore numerous creative solutions to problems, overcoming design fixation or limitation of conventional wisdom by generating alternative solutions for the designer' [34]. If there is a doubt about the computer as a design medium, it is less of an issue in performance analysis of design alternatives as it is superior to drawings and physical models in terms of accuracy, speed and representation.

\section{Conclusions}

The study concludes that the sketch, the physical model and the computer all are important methods for investigating architecture and design. Sometimes it is only a matter of difference between designers in their preference for a working method that determines which method they use rather than the objectivity of the method itself in dealing with a particular design problem. Nonetheless the logic of the computer as a mathematical system is by far more powerful than the other 
two methods in dealing with complexity, overcoming design fixation and the objective testing of 'environmental and structural performance' of design ideas. It is also more beneficial for three dimensional visualisation and design cognition. The latter issue which is sometimes called spatial visualisation 'which is the ability to mentally manipulate rotate, twist or invert pictorially presented stimulus objects' that was found to influence academic achievements of engineering students in areas such as structural design, computer aided design and engineering problem solving [35]. However, there exists a lot of misunderstanding in the literature as to what computers can and cannot do. Many scholars suggest theoretical statements about computers without themselves having the necessary skills to operate the computer to its full potential. In some cases they make wrong analogies between the computer and the human mind. For instance 'computers cannot see or dream, nor can they create: computers are language-bound. Similarly, thinkers who cannot escape the structure of language, who are unaware that thinking can occur in ways having little to do with language, are often utilizing only a small part of their brain that is indeed like a computer' [24]. It is fitting to close with a quote from Zaha Hadid who succinctly made an inclusive argument for the three working methods, when she remarked: 'I am sitting there with 15 or 20 computer screens in front of me ... it gives me yet another repertoire. You can see at the same time the section, the plan, and several moving 3D views, and in your mind you can see them in yet a different way. So I'm not sure if it weakens or strengthens your view. I just think it's a different way. We still do physical models and I still do the sketches' [36].

\section{References}

[1] Janke, R., Architectural Models, Academy Editions, London, 1978.

[2] Heath, T., Method in Architecture, John Wiley \& Sons, UK, Chapter 1, 1984.

[3] Hendrick, T. W., The Modern Architectural Model, The Architectural Press, London, p. 13, 1957

[4] Knoll, W. \& Hechinger, M., Architectural Models, Julius Hoffman Verlag GmbH, Stuttgart, pp.10-13, 1992.

[5] Frampton, K. \& Kolbowski, S., Idea as Model, Exhibition, Rizzoli International Publication Inc., New York, p. 3 \& 10, 1981

[6] Stern, R., Drawing towards a More Modern Architecture, Architectural Design, 47(6), pp. 382-383, 1977

[7] Popper, K., Objective Knowledge, Oxford University Press, p. 155, 1979.

[8] Steven, G., The Reasoning Architect, McGraw-Hill, NY, pp. 280-282, 1990.

[9] Cowan, H. et al. Models in Architecture, Elsevier Publishing, London.

[10] Zephir, A., Le Corbusier: the Philips Pavilion, Brussels, 1958 on line at http://www.lib.umd.edu/ARCH/honr219f/1958brus.html

[11] The Crypt of the Church of Colonia Guell (1898-1916), on line at http://www.gaudiclub.com/ingles/i_vida/colonia.html 
[12] Billington, D. et al., Structures Models and Architects, a Seminar in Structural Model Analysis for Arch. Students, Princeton University, p. 6, 1963.

[13] Candela. F., Understanding the Hyperbarabolic Paraboloid: Part 2, Architectural Record, August, pp. 205-215, 1958.

[14] Porter D \& Hanna R, Digital Media and Light Representation in Arch. Design, Designs on eLearning, International Conference on Teaching and Learning with Technology, University of the Arts, London, 2005.

[15] Alexander, L., Theory and Construction of Thermal Model, Sydney Common Wealth Building Station, Special Report, No 4, 1949.

[16] Barron M., Auditorium Acoustics and Architectural Design, E \& FN Spon, London, p.58, 1993.

[17] Goldschmidt, G., The Dialectics of Sketching, Creativity Research Journal, 4 (2), pp.123-143, 1991.

[18] Ziesel, J., Inquiry by Design, Cambridge University Press, p.6, 1984.

[19] Arnheim, R, Art and Visual Perception, University of California, p. vi, 1954.

[20] Arnheim, R, Visual Thinking, Faber and Faber Ltd, London, 1970.

[21] Goldschmidt, G., On Figural Conceptualisation in Architectural Design, Trappi, R, Cybernetics and Systems Research, World Scientific, Singapore, pp. 599-605, 1992.

[22] McKim, R., Experiences in Visual Thinking, Brooks/Cole, CA, USA, 1972.

[23] Mezughi, M., Architectural Drawing, Mac Journal 2, pp.93-99, 1995.

[24] Laseau, P., Graphic Thinking for Architects and Designers, Van Nostrand Reinhold Company, New York, p. 15, 1980.

[25] Evans, R., The Projective Cast, MIT Press, Chapter 3, 1995.

[26] Hanna, R., Computers and Creativity in Architectural Design, CADE 2001, Glasgow, pp. 158-166, 2001.

[27] Hanna R. \& Barber T., An Inquiry into Computers in Design: Attitudes before- Attitudes after, Design Studies, 22(3), pp 255-281, 2001.

[28] Architectural Design, New Science= New Architecture, 129, 1997.

[29] Leach N. (ed.), Designing for a digital World, Wiley Academy, UK, 2002.

[30] Domus, Visions Unfolding: Architecture in the Age of Media, 734, 1992.

[31] Galofaro, L., Digital Eisenman, Birkhauser, Basel, 1999.

[32] Gehry Partners, Architecture + Process, Thames \& Hudson, London, 1999.

[33] Steele J., Architecture and Computers, Laurence King, London, 2001.

[34] Bentley P. (ed.), Evolutionary Design by Computers, Morgan Kaufman, CA, 1999.

[35] Alias M. et al., Attitudes towards Sketching and Drawing and Spatial Visualisation in Eng. Students, International Education Journal, 3 (3), 2002.

[36] Schumacher P., Digital Hadid, Birkhaser, Basil, Switzerland, p. 11, 2004. 